\section{Ensure equal access to ancient DNA}

Research on ancient DNA relies on the availability of rare bone specimens from archaeological excavations. We consider that access to and research on these specimens should be more ethical and stringently regulated.

DNA is exceptionally well preserved in the petrous bone of the inner ear (R. Pinhasi et al. PLoS ONE 10, e0129102; 2015). Competition for these rare specimens promotes hoarding, which, along with the destruction of samples for DNA analysis, makes it hard to replicate findings. It also hinders research by scientists who are not connected to the few groups who dominate access to such samples.

Moreover, these specimens are usually exported to a few centres in Europe and the United States from countries that are not supported by costly laboratory infrastructure. This impoverishes those countries' cultural heritage through loss of scarce genetic material from local and sometimes extinct populations.

Organizations concerned with the ethics and regulation of cultural heritage and of past biological diversity need to remedy this. Ethics standards should be backed by regulations following the Nagoya Protocol (www.cbd.int/abs). A central facility to extract ancient DNA from petrous bones and to curate and store at least half of the material for replicability and accessibility purposes was recently set up in Israel (see go.nature.com/2ujxatj). Cheryl Makarewicz ChristianAlbrechts University, Kiel, Germany. Nimrod Marom, Guy Bar-Oz University of Haifa, Israel. c.makarewicz@ufg.uni-kiel.de

\section{Crypto-control your own energy supply}

Blockchains - open distributed ledgers of transactions - are emerging as an important technology for tackling community energy issues and the integration of renewable energy (see also G. Chapron Nature 545, 403-405; 2017).

Consumers are increasingly generating their own energy by investing in solar panels and micro-wind installations. In Scotland, for example, more than 300 community energy projects are in operation (go.nature.com/2vp2ypq). Blockchains operate in a decentralized way that allows individual consumers to trade energy with each other. This removes the need for an intermediary utility company, thus assuring competition is fair.

Blockchains ensure that the energy supply is traceable and that demand in one area is met by locally sourced renewable energy, where feasible. This peer-to-peer electron trading reduces carbon footprints and keeps revenues in the community. Blockchainenabled 'smart contracts' guarantee that agreements between individuals are automatically implemented.

The technology is still in its infancy. However, major challenges such as the computational cost of verifying transactions are being overcome - for example, by using algorithmic solutions to verify transactions in a distributed way. Merlinda Andoni, Valentin Robu, David Flynn Heriot-Watt University, Edinburgh, UK. m.andoni@hw.ac.uk

\section{Next decade's goals for rare diseases}

The International Rare Diseases Research Consortium (IRDiRC) has in the past six years achieved its ambitious goals for 2020 three years ahead of schedule (see Nature 472, 17; 2011). The consortium has now forged a further set of goals for 2017-27 to help the millions of people who have debilitating and lethal rare diseases.
Rare diseases were once considered medical curiosities of inscrutable phenotypic complexity and negligible public-health impact. The molecular basis of almost 6,000 rare disorders is now known. However, diagnosis of most of these conditions remains arduous, and less than $6 \%$ have approved treatments.

The new IRDiRC goals aim to achieve diagnosis within one year. Because diagnosis depends on a disorder being known, this will be accomplished through international coordination of unsolved cases. Other goals are to develop 1,000 new therapies, particularly for diseases with no approved treatment, and to create methods for assessing the impact of diagnoses and therapies on patients' well-being. (For details, see H. J.S. Dawkins et al. Clin. Transl. Sci., in the press; and C. P. Austin et al. Clin. Transl. Sci., in the press.)

The IRDiRC has nearly 50 organizations in 18 nations, with a combined yearly funding of more than US $\$ 2$ billion (see go.nature.com/2htbauh). New members are welcome to join this globally coordinated effort. Christopher P. Austin National Centre for Advancing Translational Sciences, Bethesda, Maryland, USA.

Hugh J. S. Dawkins Office of Population Health Genomics, Government of Western Australia, Perth, Australia. austinc@mail.nih.gov

\section{Taxonomy: stable taxon boundaries}

Stephen Garnett and Les Christidis argue for more governance in taxonomy to make species boundaries more stable (Nature 546, 25-27; 2017). There may be a better way to impose stability.

Taxonomy seeks natural groups by using different proxies, such as gene trees, morphology, ecology and behaviour, and testing them for strong correlations. As a result of these treatments, taxon delimitations are proposed and groups are given names according to the rules of nomenclature codes. The taxon names themselves are stable because they are defined solely by their type - such as a type specimen, a drawing of a species, or the genus that typifies a family. The application of names may, of course, be reassessed in light of new evidence.

We therefore suggest that, instead of listing and protecting species names, conservation should introduce the practice of referencing the taxonomic treatments used to apply the names to specific taxon concepts. This practice would provide stability for taxon boundaries in efforts to conserve biodiversity.

It would also boost incentives to provide and refine tools for recognizing and delimiting taxa and to deal with biodiversity beyond the category we designate as 'species. Taxonomy would be rightly recognized as a fundamental science, and conservation would have a documented scientific basis for its work - without excessive bureaucracy (see P. M. Hollingsworth Nature 546, 600; 2017).

Norbert Holstein, Federico Luebert University of Bonn, Germany. holstein@uni-bonn.de

\section{CORRECTION}

The Correspondence by E. Kowal et al. (Nature 546, 474; 2017) wrongly located the University of Pennsylvania in Pittsburgh instead of Philadelphia.

CONTRIBUTIONS

Correspondence may be sent to correspondence@ nature.com after consulting the guidelines at http:// go.nature.com/cmchno. 